\title{
Is symbolic inheritance similar to genetic inheritance?
}

Luc Steels

Computer Science Department, University of Brussels

Abstract:

Jablonka \& Lamb's book is refreshing in that it debunks the exclusively genecentered approach used these days to explain almost anything about life and human behavior. The book is very accessible and most convincing when the authors discuss biological theories of genetic and epigenetic inheritance, but it does not shy away from the more slippery terrain of behavioral and symbolic inheritance, and specifically the origins of language. But is the analogy appropriate?

The gene-centered view on language evolution simply equates genetic evolution with linguistic evolution. It argues that humans genetically inherit a language organ that determines to a large extent the structure of their language and conceptual system, and that this instinctive organ has emerged and been shaped through genetic evolution by natural selection, just like the liver. This strong nativist position is now widely disputed, both on linguistic grounds (the innate universal grammar has remained elusive even after almost 50 years of research) and on biological grounds (it is unlikely that the genome determines at such a fine-grained level the information processing of the brain). In Evolution in Four Dimensions, Jablonka \& Lamb (J\&L, 2005) survey these counterarguments and then put forward an alternative, more indirect interaction between genetic evolution and language evolution. They argue that the language system is built from neural mechanisms that are generically applicable to a wide range of cognitive functions and that the conventions of a specific language are acquired by general-purpose, socially mediated observational learning mechanisms, possibly with a slight bias established through genetic assimilation. In this scenario, the linguistic system gets copied with variation from adult individuals to the next generation, similar to the way the genome gets copied, but now by cultural instead of genetic means, thus starting a process of cultural evolution (Boyd \& Richerson 1985). What was coded as genes according to the language instinct hypothesis becomes a collection of memes transmitted through imitation (Dawkins 1975). Although I am generally in favor of the cultural hypothesis, I believe there is a major problem with this cultural transmission scenario because of the notion of information that it implies. Information is never simply there, independent of the processes that use that information. We always need to consider the physical structure, which is potentially informative, and the interpreter, who brings out the information and uses it in a specific context. For example, the information in a computer program (which is, after all, just a sequence of switch settings in a computer's memory) only becomes information when it is interpreted by a specific compiler and an operating system, using the primitive machine instructions that the computer can perform. The exact behavior of a running program depends in addition on the specific inputs and prior information states present at the time of program execution. If none of this is right, the program is totally useless and does not run at all. Similarly, the DNA macromolecule only becomes information when there is the interpretive machinery that can act upon it. 
Otherwise, it is a totally dead piece of matter. Just like a computer program, the interpreting process integrates the environment. So in that sense, the genetic system is not just the replicating DNA; it necessarily requires and includes the epigenetic system. Likewise, aspects of a situation in the world or of the behavior of another individual only become informative after an enormously complex process of perception and context-sensitive interpretation has been able to make sense of what is going on. A collection of speech sounds only becomes a carrier of information if there is somebody that can parse and interpret it in a specific context. The cultural transmission scenario assumes that enough information is present in the perception of behaviors or in language sentences so that the system needed to interpret and reuse them can be copied by imitation from one individual to another. Here lies the difficulty. The imitator/learner must have a sense of what aspects of reality are relevant and what the underlying intentions are before he or she can imitate. The real world is infinitely complex. Without an interpretive capacity in place, the imitator cannot know what exactly needs to be retained in his or her own behavior and when it is appropriate. For example, the tones of a vowel are very relevant in Chinese but are irrelevant in English, so an English speaker trying to imitate Chinese will not properly pay attention to the tonal distinctions, let alone be capable to replicate them. This is the fundamental paradox for all models of behavioral or symbolic inheritance that rely on imitation or observational learning - to imitate, you must know what counts as information and what is the intention of the producer. So the behavioral or cultural transfer of the interpretive capacity must already have taken place before the imitative act. This is in contrast to the copying mechanism underlying genetic inheritance, which does not need to know anything about what it is copying. This paradox explains why attempts to operationalize imitation in artificial systems have failed, despite a lot of effort (Dautenhahn \& Nehaniv 2002). Models of cultural evolution based on imitation appear to assume what they try to explain. So what is the alternative? Perhaps it is not such a good idea to make the analogy between different forms of inheritance so strong. Szathmáry (2006) draws our attention to a distinction between replicators and reproducers that may be helpful here: Replicators multiply with heredity plus variation, so that selection can act on the population of variants. However, replicators cannot replicate on their own. To copy them, a reproducer is needed - an entity that can do the replication. Genes are replicators but not reproducers; the cell is the reproducer. But because the cell can perform replication of genes, which can then reproduce another cell given the right additional (epigenetic) context, they can also be regarded as replicators. Viruses, in contrast, are replicators but not reproducers themselves, because they need another living cell to reproduce. This indicates the following analogies and differences between genetic inheritance and symbolic or behavioral inheritance (Steels 2004): Utterances or features of utterances can be viewed as replicators (as in Croft 2000). Every time the same sort of utterance (or feature of an utterance) is produced, it is a replication, unavoidably, with some variation. The reproducer is the speaker's total language system, which might have had to be expanded to achieve the speaker's communicative goals for that utterance. When the utterance is interpreted by the hearer, he or she will have to exercise his or her own total language system, possibly expanding or adjusting it as well. 
Within this scenario, language systems are not transferred by imitation or observational learning but are actively constructed by speakers and hearers and are aligned to maximize success in communication. With enough interactions, the language system of a speaker will seem to have been transmitted to the hearer; but in fact, the transmission does not at all take place by copying, the way it does for DNA. 